Ciência Florestal, Santa Maria, v. 22, n. 1, p. 171-181, jan.-mar., 2012

ISSN 0103-9954

\title{
EFEITO DOS SÓLIDOS DISSOLVIDOS DA MADEIRA NA BRANQUEABILIDADE E NAS PROPRIEDADES FÍSICO-MECÂNICAS E ÓPTICAS DE POLPA KRAFT BRANQUEADA DE EUCALIPTO
}

\author{
EFFECT OF WOOD DISSOLVED SOLIDS ON BLEACHABILITY AND PHYSICAL, \\ MECHANICAL AND OPTICAL PROPERTIES OF EUCALYPTUS BLEACHED PULP
}

\author{
Dalton Longue Júnior ${ }^{1}$ Jorge Luiz Colodette ${ }^{2}$
}

\begin{abstract}
RESUMO
Muitos estudos demonstram que o rendimento da polpação kraft pode ser melhorado pela readsorção de xilanas à polpa durante o cozimento, e o licor negro kraft contém fração significativa das xilanas da madeira, sendo uma importante fonte dessas macromoléculas. Porém, o efeito dessa técnica nas propriedades físicomecânicas e ópticas de polpa branqueada de eucalipto, ainda não está bem esclarecido. Neste estudo foram produzidas polpas de eucalipto provenientes de cozimento kraft convencional, com diferentes adições de licor negro $(0,25,50,75$ e $100 \% \mathrm{v} / \mathrm{v})$. Os cozimentos foram conduzidos até número kappa 17-18 e as polpas resultantes foram deslignificadas com oxigênio em condições fixas. O branqueamento foi realizado utilizando uma sequência $\mathrm{D}_{\mathrm{HT}}(\mathrm{EP}) \mathrm{DP}$ até alvura final $92 \%$ ISO. Em seguida as polpas foram refinadas num moinho PFI a diferentes níveis de revoluções $(0,500,1.500,3.000$ e 6.000$)$ e realizados testes de resistência ao rasgo, ao arrebentamento e à passagem de ar, alongamento, opacidade, densidade, volume específico aparente, capilaridade Klemm e índice de retenção de água (WRV). A eficiência e o ganho de alvura da pré-deslignificação com oxigênio foram ligeiramente prejudicados pela adição de licor negro ao cozimento kraft. A adição de $50 \%$ de licor negro aos cozimentos resultou em polpas de pior branqueabilidade, sendo a demanda de cloro ativo aumentada em $5,3 \mathrm{~kg} / \mathrm{t}$ celulose seca para obtenção de alvura 92 \% ISO. As propriedades físico-mecânicas e ópticas das polpas kraft branqueadas não foram influenciadas, significativamente, pelo uso de licor negro no cozimento.
\end{abstract}

Palavras-chave: remoção de hemiceluloses; xilanas; branqueamento; propriedades físico-mecânicas.

\begin{abstract}
Many studies have shown that the yield of kraft pulping can be improved by xylan reabsorption to the pulp during the cooking, and kraft black liquor contains significant fraction of xylan from wood, an important source of these macromolecules. However, the effect of this technique in the physical-mechanical and optical properties of bleached eucalyptus pulp is not very clear. This study produced pulp from eucalyptus kraft conventional cooking with different additions of black liquor $(0,25,50,75$ and $100 \% \mathrm{v} / \mathrm{v})$. The cooking was conducted to kappa number 17-18 and the resulting pulps were delignified with oxygen in fixed conditions. Bleaching was performed using a sequence $\mathrm{D}_{\mathrm{HT}}(\mathrm{EP}) \mathrm{DP}$ to end whiteness $92 \%$ ISO. Next pulps were refined in a PFI mill at different levels of revolutions $(0,500,1,500,3,000$ and 6,000) and tests of tear resistance, bursting and the air flow, elongation, opacity, density, apparent specific volume, Klemm capillary and water retention value (WRV). The efficiency and brightness gain of the pre-delignification were slightly affected by the addition of black liquor in kraft pulping. The addition of $50 \%$ of black liquor to cooking resulted in a worse pulp ability for bleaching, and the chlorine demand increased by $5.3 \mathrm{~kg} / \mathrm{t}$ pulp oven dry to obtain $92 \%$ ISO brightness. The physical-mechanical and optical properties bleached kraft pulps were not influenced significantly by the use of black liquor in cooking.
\end{abstract}

Keywords: hemi-cellulose removal; xylans; bleaching; physical-mechanical properties.

1. Engenheiro Florestal, Doutorando do Programa de Pós-graduação em Ciência Florestal, Centro de Ciências Agrárias, Universidade Federal de Viçosa, Av. P.H.Rolfs, s/n, CEP 36570-000, Viçosa (MG). Bolsista do CNPq. daltonufv@yahoo.com

2. Engenheiro Florestal, Dr., Professor Titular do Departamento de Engenharia Florestal, Universidade Federal de Viçosa, Av. P.H.Rolfs, s/n, CEP 36570-000, Viçosa (MG). colodett@ufv.br

Recebido para publicação em 23/04/2010 e aceito em 17/03/2011 


\section{INTRODUÇÃO}

O processo de cozimento kraft tem como premissa a individualização das fibras da madeira pela remoção da lignina. Para que isso ocorra, uma grande fração de carboidratos é dissolvida por reações químicas nas cadeias de celulose e hemiceluloses. As hemiceluloses são os carboidratos mais degradados durante o cozimento, devido à sua maior reatividade quando comparadas com a celulose e, por isso, têm recebido muita atenção, pois sua maior preservação significaria ganhos apreciáveis de rendimento (GULLICHSEN e PAULAPURO, 1999). O destino das hemiceluloses removidas da madeira é o licor negro kraft. Portanto, a recuperação das hemiceluloses contidas nesse licor pode resultar em significativo aumento do rendimento da polpação kraft.

As hemiceluloses, além de afetar o rendimento de produção da polpa, desempenham importantes funções no processamento e nas propriedades da polpa e do papel (BRITT, 1970), pois podem afetar importantes interações químicas das fibras com a água e com outros produtos químicos que são incorporados para a formação do papel.

Nesse contexto, alterações no cozimento, como adição de diferentes quantidades de licor negro, influenciam o branqueamento e podem interferir nas propriedades finais das fibras, mesmo após a refinação.

O objetivo deste estudo foi avaliar o impacto da readsorção de hemiceluloses, por meio da reutilização de quantidades elevadas de licor negro no processo kraft convencional, assim como avaliar o impacto desta prática na branqueabilidade, na refinabilidade e nas propriedades ópticas e físicomecânicas da polpa resultante.

\section{MATERIAL E MÉTODO}

Para produção de polpa marrom, foram usados $500 \mathrm{~g}$ de cavacos industriais de Eucalyptus grandis. Esses cavacos foram submetidos a cozimentos kraft convencionais, com diferentes adições de licor negro $(0,25,50,75$ e $100 \% \mathrm{v} / \mathrm{v})$, numa relação licor/madeira de $4: 1 \mathrm{~m}^{3} / \mathrm{t}$. A temperatura de cozimento foi de $170^{\circ} \mathrm{C}$, o tempo até a temperatura foi de 90 minutos e o tempo à temperatura de mais 40 minutos, a sulfidez utilizada foi de $35 \%$ e as cargas de álcali foram otimizadas para um kappa 17-18.

\section{Pré-branqueamento}

As polpas marrons foram então

deslignificadas com oxigênio, em condições fixas para todos os tratamentos: temperatura $=103^{\circ} \mathrm{C}$; tempo $=44 \mathrm{~min} ;$ pressão $=366 \mathrm{kPa}$; consistência $=10$ $\%$; carga de $\mathrm{O}_{2}, 18,0 \mathrm{~kg} / \mathrm{t}$ seca ao ar; carga de $\mathrm{NaOH}$, $22,1 \mathrm{~kg} / \mathrm{t} \mathrm{seca}$ ao ar.

A pré-deslignificação com oxigênio foi efetuada em reator/misturador modelo Mark V (Quantum Technologies Inc.) com amostras de $300 \mathrm{~g}$ de polpa absolutamente secas. A polpa foi depositada no reator na consistência adequada e aquecida até a temperatura desejada. Atingida a temperatura, e sob efeito de agitação constante, foram injetadas as cargas preestabelecidas de $\mathrm{NaOH}$ ao sistema, elevando-se a pressão de reação com oxigênio até o valor desejado. Terminado o tempo total de reação, foram extraídas amostras do licor residual, para análises de $\mathrm{pH}$. A polpa foi transferida para o descarregador de polpa e, então, lavada com o equivalente a $9 \mathrm{~m}^{3}$ de água destilada por tonelada de polpa.

\section{Branqueamento}

$\mathrm{O}$ branqueamento das polpas foi realizado com a sequência $\mathrm{D}_{\mathrm{HT}}(\mathrm{EP}) \mathrm{DP}$. As condições gerais do branqueamento estão descritas na Tabela 1.

Ao final de cada estágio de branqueamento a polpa foi lavada com o equivalente a $9 \mathrm{~m}^{3}$ de água morna destilada por tonelada de polpa. E todos os estágios foram realizados em duplicata.

\section{Deslignificação com dióxido de cloro a quente - $\mathbf{D}_{\mathrm{HT}}$}

Este estágio foi realizado em saco de polietileno em banho-maria. A amostra foi misturada com água e aquecida a $90^{\circ} \mathrm{C}$. À amostra aquecida foi adicionada e misturada uma quantidade desejada de dióxido de cloro. Neste momento a temperatura diminuiu até o valor desejado. Logo em seguida, o $\mathrm{pH}$ inicial foi medido, o saco selado hermeticamente e colocado em um banho de vapor na temperatura desejada. Após a conclusão do tempo de reação, uma amostra de $300 \mathrm{~mL}$ de licor foi coletada para análise de $\mathrm{pH}$ e do residual de cloro.

\section{Extração oxidativa com peróxido - EP}

Este estágio foi realizado em reator/ misturador Quantum Mark, modelo V. As cargas de hidróxido de sódio e de peróxido de hidrogênio foram adicionadas no reator depois que a temperatura foi alcançada, e nesse momento o $\mathrm{pH}$ inicial foi medido. Ao final da reação, a polpa foi transferida a um recipiente e $200 \mathrm{~mL}$ de licor foram coletados para análises de $\mathrm{pH}$ e residuais de peróxido. 
TABELA 1: Condições gerais de branqueamento.

TABLE 1: General conditions of pulping bleaching.

\begin{tabular}{|c|c|c|c|c|}
\hline Condições & $\mathrm{D}_{\mathrm{HT}}$ & $(\mathrm{EP})$ & $\mathrm{D}$ & $\mathrm{P}$ \\
\hline Consistência (\%) & 7,5 & 10 & 10 & 10 \\
\hline Tempo (min.) & 197 & 103 & 247 & 66 \\
\hline Temperatura $\left({ }^{\circ} \mathrm{C}\right)$ & 93 & 89 & 66 & 90 \\
\hline $\mathrm{ClO}_{2}$ como $\mathrm{Cl}_{2}(\mathrm{~kg} / \mathrm{tas} *)$ & 13,5 & - & $3 ; 6 ; 9$ e 13,7 & - \\
\hline $\mathrm{H}_{2} \mathrm{O}_{2}\left(\mathrm{~kg} / \mathrm{tas}^{*}\right)$ & - & 2,3 & & 2,7 \\
\hline $\mathrm{pH}$ final & 2,5 & 11,0 & 4,5 & 11,0 \\
\hline
\end{tabular}

Em que: $*$ tas $=$ tonelada de celulose seca absolutamente seca

\section{Branqueamento com dióxido de cloro - $\mathrm{D}_{1}$}

Este estágio foi realizado em saco de polietileno em banho-maria. As amostras de polpa foram misturadas completamente com água, dióxido de cloro e hidróxido de sódio, ou ácido sulfúrico para o controle do $\mathrm{pH}$, e em seguida foram lacradas hermeticamente, pré-aquecidas à temperatura desejada em um forno de micro-ondas e colocadas em um banho de vapor, mantido na temperatura constante. Depois de terminado o tempo de reação, uma amostra de $40 \mathrm{~mL}$ de licor foi coletada para análise de $\mathrm{pH}$ e análises de residual de dióxido de cloro.

\section{Branqueamento com peróxido de hidrogênio - $\mathbf{P}$} Este estágio foi realizado em saco de polietileno em banho-maria. As amostras de polpa foram misturadas completamente com água, peróxido de hidrogênio e hidróxido de sódio, para o controle do $\mathrm{pH}$, e em seguida foram lacradas hermeticamente, pré-aquecidas à temperatura desejada em um forno de micro-ondas e colocadas em um banho de vapor mantido na temperatura constante. Depois de terminado o tempo de reação, uma amostra de $40 \mathrm{~mL}$ de licor foi coletada para análise de $\mathrm{pH}$ e análises de residual de peróxido de hidrogênio.

Testes físico-mecânicos e ópticos das polpas kraft As polpas foram refinadas em moinho PFI, conforme norma TAPPI wd-97, com diferentes níveis de revoluções $(0,500,1.500,3.000$ e 6.000). Dessas amostras foram formadas folhas manuais com gramatura de aproximadamente $60 \mathrm{~g} / \mathrm{m}^{2}$, em uma Coluna Formadora TAPPI, fabricada pela REGMED.

Os testes relacionados a esforços de tração foram feitos em aparelho Instron, com distância entre garras de $100 \mathrm{~mm}$, velocidade de teste de 25 $\mathrm{mm} / \mathrm{min}$ e célula de carga de $1.000 \mathrm{~N}$.

Os testes de resistência ao rasgo, ao arrebentamento e à passagem de ar foram realizados em aparelhos Eldendorf, Müllen e porosímetro Gurley, respectivamente.

\section{Análises da polpa}

As polpas kraft não branqueadas e prédeslignificadas com oxigênio foram então submetidas a análises de número kappa, viscosidade e alvura.

As metodologias utilizadas durante todo o estudo se encontram na Tabela 2.

\section{Análises estatísticas}

As análises estatísticas iniciais dos experimentos (pré-branqueamento e branqueamento) foram feitas com o auxilio dos softwares Statistica 6.0 e Microsoft Office Excel 2003. Foram feitas análises de variância (ANOVA) e testes de comparação de médias (Tukey), usando um nível de significância de $5 \%$.

Para análise dos resultados finais dos testes físico-mecânicos das polpas tratadas com diferentes adições de licor negro em diferentes níveis de refino $(0,500,1500,3000$ e 6000 revoluções) foi utilizado o software SAS - STATISTICAL ANALISYS SYSTEM (2000). Os dados obtidos com a realização do refino foram analisados estatisticamente por meio de análise de regressão. As equações ajustadas foram comparadas pelo teste $\mathrm{F}$, utilizando-se o teste de identidade de modelos e adotando-se um nível de significância de até $5 \%$ de probabilidade, conforme metodologia apresentada por Regazzi (1993) para modelos lineares e Regazzi e Silva (2004) para modelos não lineares. 
TABELA 2: Procedimentos analíticos para caracterização físico-química da madeira, licores e polpa celulósica.

TABLE 2: Analytical proceedings to physical chemical characterization of the wood, liquors and cellulose pulp.

\begin{tabular}{ll}
\hline \multicolumn{1}{c}{ Parâmetros } & \multicolumn{1}{c}{ Procedimentos } \\
\hline Número kappa & Tappi T 236 cm-85 \\
Viscosidade & Tappi T230 om-94 \\
Formação manual de folhas & Tappi T 218 sp-97 \\
Alvura & Tappi T525 om-92 \\
Reversão de alvura & Tappi UM 200 \\
Análises de soluções e residuais de dióxido de & McDonald, R.G., 1967 \\
cloro e peróxido de hidrogênio & \\
Índice de rasgo & ISO1974:90 \\
Índice de estouro & ISO 2759:83 \\
Índice de tração & ISO 1924/2:94 \\
Alongamento & ISO 1924/2 \\
Opacidade & ISO 2471:98 \\
Resistência à passagem de ar & ISO 5636/5:86 \\
Densidade & ISO 534:05 \\
Volume específico aparente & ISO 534:05 \\
Capilaridade Klemm & ISO 8787:86 \\
Índice de retenção de água (WRV) & TAPPI UM 256 \\
Grau Shopper-Riegler & ISO 5267/1, ABTCP C 10 \\
\hline
\end{tabular}

\section{RESULTADOS E DISCUSSÃO}

Desempenho da pré- $\mathrm{O}_{2}$ para polpas derivadas de cozimentos $k$ raft com adição de licor negro

A pré-deslignificação com oxigênio $\left(\right.$ Pré- $\left.\mathrm{O}_{2}\right)$ foi realizada sob condições fixas, para todas as polpas derivadas de cozimentos, com diferentes adições de licor negro. Os resultados mostram que a eficiência da pré- $\mathrm{O}_{2}$ para polpa kraft preparada com adição de licor negro foi igual estatisticamente até adição de $75 \%$ desse licor, quando houve melhora do grau de deslignificação (Figura 1).

Pela análise de variância a $5 \%$ de significância, a eficiência de deslignificação apresentou diferenças estatísticas $\left(\mathrm{F}_{\text {calc }} 22,8-\mathrm{F}_{\text {tab }}\right.$ 3,48 ); o número kappa da polpa marrom não apresentou diferenças estatísticas, pois os cozimentos foram realizados propositalmente a um mesmo grau de deslignificação ( $\left.\mathrm{F}_{\text {calc }} 3,4-\mathrm{F}_{\text {tab }} 4,76\right)$; e o número kappa da polpa pré- $\mathrm{O}_{2}\left(\mathrm{~F}_{\text {calc }} 49,5-\mathrm{F}_{\text {tab }} 3,48\right)$ também apresentou diferenças estatísticas. Os resultados do teste de Tukey se encontram na Tabela 3.

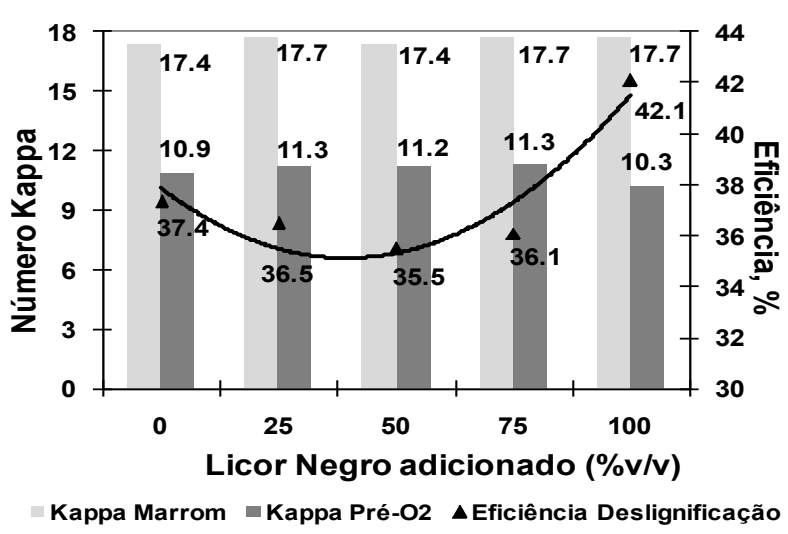

FIGURA 1: Eficiência da pré-O $\mathrm{O}_{2}$ para polpas (número kappa 17-18) derivadas de cozimento kraft com diferentes adições de licor negro.

FIGURE 1: Pre- $\mathrm{O}_{2}$ efficiency for pulps (kappa number 17-18) from kraft cooking with different additions of black liquor. 
TABELA 3: Resumo dos testes de Tukey para os parâmetros da pré-deslignificação com oxigênio das polpas oriundas de cozimentos kraft com diferentes concentrações de licor negro.

TABLE 3: Summary of Tukey tests for the parameters of pre-delignification of pulp from kraft cooking with different concentrations of black liquor.

\begin{tabular}{|c|c|c|c|c|c|}
\hline \multirow{2}{*}{ Parâmetros } & \multicolumn{5}{|c|}{ Adição de Licor Negro (\% v/v) } \\
\hline & 0 & 25 & 50 & 75 & 100 \\
\hline Eficiência da pré-O ${ }_{2}(\%)$ & $37,5 \mathrm{a}$ & $36,4 \mathrm{a}$ & $36,4 \mathrm{a}$ & $35,4 \mathrm{a}$ & $42,6 \mathrm{~b}$ \\
\hline Número kappa (polpa marrom) & $17,4 \mathrm{a}$ & $17,7 \mathrm{a}$ & $17,4 \mathrm{a}$ & $17,7 \mathrm{a}$ & $17,7 \mathrm{a}$ \\
\hline Número kappa (polpa pré- $\mathrm{O}_{2}$ ) & $10,9 \mathrm{~b}$ & $11,3 \mathrm{c}$ & $11,2 \mathrm{c}$ & $11,3 \mathrm{c}$ & $10,3 \mathrm{a}$ \\
\hline Seletividade & $0,22 \mathrm{a}$ & $0,23 \mathrm{a}$ & $0,24 a b$ & $0,23 \mathrm{a}$ & $0,27 b$ \\
\hline Viscosidade (cP, polpa marrom) & $61,1 \mathrm{a}$ & $58,4 \mathrm{~b}$ & $54,3 \mathrm{c}$ & $52,9 \mathrm{~cd}$ & $51,3 \mathrm{e}$ \\
\hline Viscosidade (cP, polpa pré- $\mathrm{O}_{2}$ ) & $31,8 \mathrm{a}$ & $21,9 \mathrm{~b}$ & $28,2 \mathrm{c}$ & $25,4 \mathrm{~d}$ & $23,4 \mathrm{e}$ \\
\hline Ganho de alvura (\%) & $14,7 \mathrm{~b}$ & $13,2 \mathrm{a}$ & $13,5 \mathrm{a}$ & $15,6 \mathrm{c}$ & $15,7 \mathrm{c}$ \\
\hline Alvura, \%ISO (polpa marrom) & $40,8 \mathrm{a}$ & $39,5 \mathrm{~b}$ & $38,9 \mathrm{c}$ & $37,5 \mathrm{c}$ & $34,0 \mathrm{~d}$ \\
\hline Alvura, \%ISO (polpa pré- $\mathrm{O}_{2}$ ) & $55,4 \mathrm{a}$ & $52,7 \mathrm{~b}$ & $52,4 \mathrm{~b}$ & $53,1 \mathrm{~b}$ & $49,7 \mathrm{c}$ \\
\hline
\end{tabular}

Deve ser ressaltado que, em todos os casos, os números kappa após a deslignificação com oxigênio ficaram abaixo de 12 , valor tido como limite superior na maioria das fábricas que trabalham com polpa de kappa marrom 17-18. Dessa forma, a pré- $\mathrm{O}_{2}$ conseguiu diminuir o número kappa das polpas a níveis aceitáveis, em todos os casos.

Verifica-se ainda na Figura 1 que os valores de número kappa, após a pré- $\mathrm{O}_{2}$ variaram de 10,9 a 11,3 , para níveis de adição de licor negro de até 75 \%. Para o nível de adição de 100 \% de licor negro, o número kappa foi reduzido para 10,3, indicando maior eficiência da pré- $\mathrm{O}_{2}$ para a polpa produzida nesta condição. Esse resultado pode ser explicado pelo elevado conteúdo de lignina lixiviável em álcali presente na polpa produzida com excesso de licor negro, a qual é facilmente removida na pré- $\mathrm{O}_{2}$.

Como mostrado na Figura 2, a seletividade da pré- $\mathrm{O}_{2}$ aumentou, significativamente, à medida que foi adicionado licor negro ao cozimento, devendo ser ressaltado que, até a adição de $75 \%$ de licor negro, não houve diferença estatística, como indicado no teste de Tukey (Tabela 3).

A seletividade da pré- $\mathrm{O}_{2}$ é muito influenciada pelo valor de viscosidade da polpa marrom, sendo verificado que o processo menos seletivo aconteceu para polpas marrons de alta viscosidade. Essa tendência é muito visível na Figura 2.

Foram verificadas diferenças significativas para as viscosidades das polpas marrons $\left(\mathrm{F}_{\text {calc }}\right.$ $\left.69,1 \mathrm{e}_{\text {tab }}-3,48\right)$ e pré- $\mathrm{O}_{2}\left(\mathrm{~F}_{\text {calc }}-123,3\right.$ e $\left.\mathrm{F}_{\text {tab }}-3,48\right)$, conforme Tabela 3. A redução da viscosidade devido à adição de licor negro ao cozimento kraft pode ser compreendida pela maior carga de álcali utilizada nos cozimentos à medida que a adição desse licor foi aumentada. Também a readsorção de xilanas contidas nesse licor negro, ou seja, maior presença de carboidratos de menor grau de polimerização, contribuiu para reduzir a viscosidade das polpas.

Tanto os valores de ganho de alvura $\left(\mathrm{F}_{\text {calc }}-\right.$ 35,6 e $\mathrm{F}_{\text {tab }}-3,48$ ), quanto os de alvura das polpas antes da pré- $\mathrm{O}_{2}\left(\mathrm{~F}_{\text {calc }}-566,6\right.$ e $\left.\mathrm{F}_{\text {tab }}-3,48\right)$ e após

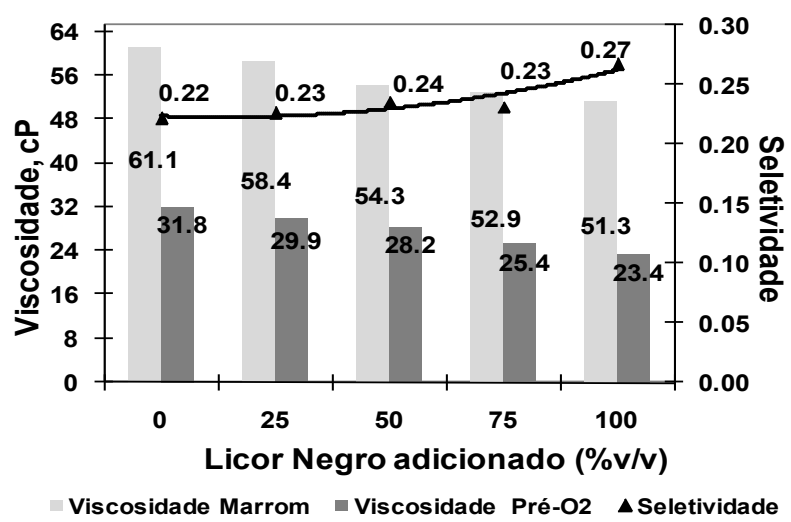

FIGURA 2: Seletividade da pré-O $\mathrm{O}_{2}$ para polpas (número kappa 17-18) derivadas de cozimento kraft com diferentes adições de licor negro.

FIGURE 2: Pre- $\mathrm{O}_{2}$ selectivity for pulps (kappa number 17-18) from kraft cooking with different additions of black liquor. 
pré- $\mathrm{O}_{2}\left(\mathrm{~F}_{\text {calc }}-118,3\right.$ e $\left.\mathrm{F}_{\text {tab }}-3,48\right)$, apresentaram diferenças significativas pelo teste de Tukey (Tabela 3).

$\mathrm{O}$ ganho de alvura na pré- $\mathrm{O}_{2}$ diminuiu, significativamente, com a adição de até $50 \%$ de licor negro (Figura 3).

À medida que se adicionou licor negro aos cozimentos verificou-se redução da alvura da polpa marrom e da polpa pré-O ${ }_{2}$ (Figura 3). Esse fato pode ser explicado pela readsorção sobre a polpa de xilanas, contendo fragmentos de lignina. As xilanas dissolvidas no licor negro podem estar associadas à lignina através dos complexos lignina-carboidratos. Uma observação importante é que a menor alvura conseguida após a pré- $\mathrm{O}_{2}$, que foi de $49,7 \%$ ISO para adição de $100 \%$ de licor negro, é ainda suficiente para a maioria das aplicações industriais.

\section{Desempenho do branqueamento para polpas derivadas da polpação kraft com adição de licor negro}

Um resumo dos resultados do branqueamento das polpas oriundas de cozimento kraft com diferentes adições de licor negro se encontra na Tabela 4.

Com o aumento da adição de licor negro aos cozimentos, verificou-se maior necessidade de cloro ativo total no branqueamento para se atingir alvura $92 \%$ ISO. O aumento da demanda de $\mathrm{ClO}_{2}$ chegou a $26 \%$, quando foi adicionado $50 \%$ de licor negro aos cozimentos. Esse aumento corresponde a $0,53 \%$ a mais de cloro ativo total, ou seja, $5,3 \mathrm{~kg} / \mathrm{t}$ de cloro ativo total. Segundo Sjödahl (2006), o consumo de dióxido

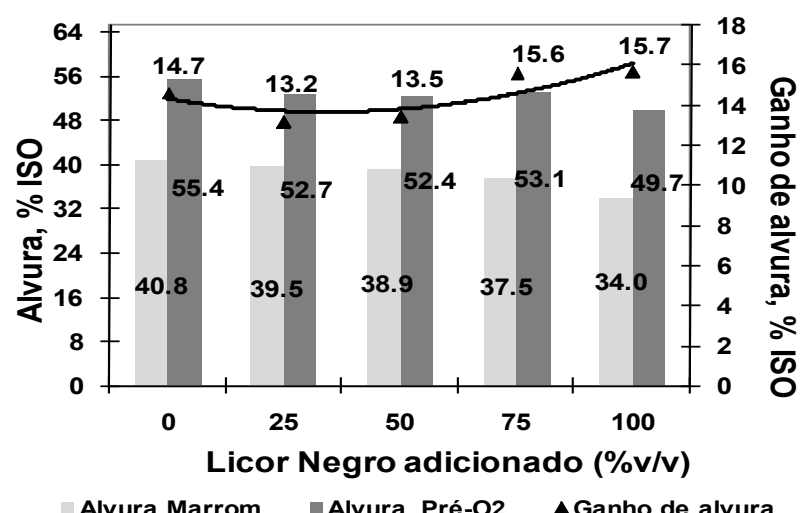

FIGURA 3: Ganho de alvura da pré- $\mathrm{O}_{2}$ para polpas (número kappa 17-18) derivadas de cozimento kraft com diferentes adições de licor negro.

FIGURE 3: Pre- $\mathrm{O}_{2}$ gain whiteness for pulps (kappa number 17-18) from kraft cooking with different additions of black liquor. de cloro foi aumentado em $13 \%$, o equivalente a $5 \mathrm{~kg}$ de cloro ativo total por tonelada de polpa branqueada, quando o licor negro foi adicionado ao cozimento de madeira de coníferas.

$\mathrm{O}$ custo de químicos do branqueamento aumentou à medida que o licor negro foi adicionado aos cozimentos $\left(\mathrm{F}_{\text {calc }}-450,0\right.$ e $\left.\mathrm{F}_{\text {tab }}-5,19\right)$. Esse custo teve aumento mais acentuado para adição de $100 \%$ de licor negro (Figura 4). Os resultados do teste de Tukey para o custo do branqueamento se encontram na Tabela 4.

Segundo Sjödahl (2006), em um estudo utilizando polpa kraft de coníferas, a adição de compostos dissolvidos de madeira aumenta o coeficiente de absorção de luz da polpa (k), a $457 \mathrm{~nm}$, tornando-a mais escura.

Os valores de branqueabilidade, ou seja, o consumo de reagentes químicos por unidade de número kappa removido das polpas durante o branqueamento, foram estatisticamente significativos $\left(\mathrm{F}_{\text {calc }}-408,9\right.$ e $\left.F_{\text {tab }}-5,19\right)$. Dessa forma, a adição de licor negro decresce a branqueabilidade das polpas, sendo mais crítico para adição de $100 \%$ de licor negro (Figura 4). Segundo Sjödahl (2006), polpas kraft de coníferas de número kappa 25 , produzidas com adição de licor negro, apresentaram baixa branqueabilidade quando comparadas às polpas produzidas sem adição de licor negro. $\mathrm{O}$ teste de Tukey para a branqueabilidade se encontra na Tabela 4. Em um estudo realizado com cargas fixas dos reagentes de branqueamento, para todas as amostras de polpa, ficou claramente evidenciada a menor branqueabilidade das polpas oriundas de cozimentos com adição de licor negro. A alvura final da polpa branqueada foi significativamente reduzida $\left(\mathrm{F}_{\text {calc }}-59,1\right.$ e $\left.\mathrm{F}_{\text {tab }}-5,19\right)$ à medida que o licor negro foi adicionado aos cozimentos (Figura 5). A alvura da polpa oriunda do cozimento com $100 \%$ de licor negro foi $2 \%$ ISO menor que àquela da polpa-referência. As diferenças entre os tratamentos são evidenciadas pelo teste de Tukey e se encontram na Tabela 4.

A análise de reversão de alvura foi realizada por meio do número de cor posterior (NCP). Essa medição permite avaliar, mais precisamente, a reversão de alvura, pois possibilita comparar polpas com diferentes níveis de alvura final, como foi o caso deste estudo. Os valores de NCP foram muito baixos para todas as polpas, variando entre a faixa de 0,132 a 0,178 (Figura 5). Não foi verificada nenhuma diferença significativa entre os tratamentos, mostrando que mesmo as adições elevadas de licor negro aos cozimentos não afetaram significativamente a reversão de alvura $\left(\mathrm{F}_{\text {calc }}-5,0\right.$ e $\left.\mathrm{F}_{\text {tab }}-5,19\right)$. 
TABELA 4: Resultados do branqueamento das polpas oriundas dos cozimentos kraft com diferentes adições de licor negro, pela sequência $\mathrm{OD}_{\mathrm{HT}}(\mathrm{EP}) \mathrm{DP}$.

TABLE 4: Results of pulp bleaching from the kraft cooking with different additions of black liquor, by the sequence $\mathrm{OD}_{\mathrm{HT}}(\mathrm{EP}) \mathrm{DP}$.

\begin{tabular}{|c|c|c|c|c|c|}
\hline \multirow{2}{*}{ Resultados } & \multicolumn{5}{|c|}{ Adição de Licor Negro (\% v/v) } \\
\hline & 0 & 25 & 50 & 75 & 100 \\
\hline $\mathrm{ClO}_{2}\left(\%\right.$ como $\left.\mathrm{Cl}_{2}\right)$ & 1,99 & 2,50 & 2,53 & 2,23 & 3,00 \\
\hline $\mathrm{H}_{2} \mathrm{O}_{2}(\%)$ & 0,50 & 0,50 & 0,50 & 0,50 & 0,50 \\
\hline $\mathrm{O}_{2}(\%)$ & 1,82 & 1,82 & 1,82 & 1,82 & 1,82 \\
\hline $\mathrm{NaOH}(\%)$ & 3,92 & 3,92 & 3,92 & 3,92 & 3,92 \\
\hline $\mathrm{H}_{2} \mathrm{SO}_{4}(\%)$ & 1,72 & 1,40 & 1,40 & 1,40 & 1,40 \\
\hline $\mathrm{ClO}_{2}\left(\%\right.$ como $\left.\mathrm{ClO}_{2}\right)$ & 0,76 & 0,95 & 0,96 & 0,85 & 1,14 \\
\hline${ }^{1 /}$ Cloro ativo total $(\%)$ & 3,03 & 3,53 & 3,57 & 3,26 & 4,03 \\
\hline Branqueabilidade ( $\%$ cloro ativo $/ \Delta \mathrm{K}$ ) & $3,24 \mathrm{a}$ & $3,03 \mathrm{c}$ & $2,97 \mathrm{c}$ & $3,12 \mathrm{~b}$ & $2,53 \mathrm{~d}$ \\
\hline 2/ Custo de químicos, US\$/t a.s. & $34,6 \mathrm{a}$ & $36,3 \mathrm{c}$ & $36,4 \mathrm{c}$ & $35,2 \mathrm{~b}$ & $38,2 \mathrm{~d}$ \\
\hline${ }^{3 / A}$ lvura final (\% ISO) & 92,1 & 92,3 & 92,4 & 92,4 & 92,0 \\
\hline Alvura revertida (\% ISO) & 90,7 & 90,5 & 91,0 & 91,0 & 90,6 \\
\hline Reversão de alvura (\% ISO) & 1,4 & 1,8 & 1,4 & 1,4 & 1,4 \\
\hline${ }^{4 / A l v u r a ~ f i n a l ~(\% ~ I S O) ~}$ & $92,0 \mathrm{a}$ & $91,0 \mathrm{bc}$ & $90,2 \mathrm{~d}$ & $91,4 \mathrm{~b}$ & $90,9 \mathrm{c}$ \\
\hline Número de cor posterior (NCP) & $0,138 \mathrm{a}$ & $0,178 \mathrm{a}$ & $0,132 \mathrm{a}$ & $0,132 \mathrm{a}$ & $0,140 \mathrm{a}$ \\
\hline Viscosidade da polpa (mPas) & $11,7 \mathrm{a}$ & $10,9 \mathrm{a}$ & $11,8 \mathrm{a}$ & $10,8 \mathrm{a}$ & $11,1 \mathrm{a}$ \\
\hline${ }^{5} /$ Rendimento pré- $\mathrm{O}_{2}(\%)$ & 97,7 & 97,8 & 97,9 & 97,7 & 97,7 \\
\hline
\end{tabular}

Em que: ${ }^{1 /}$ Cloro ativo total $=\left(\mathrm{ClO}_{2} * 2,63+\mathrm{H}_{2} \mathrm{O}_{2} * 2,09\right) ;{ }^{2 /}$ Calculado para alvura de $92,0 \%$ ISO, exatamente; ${ }^{3 /}$ Alvura final objetivo $92 \%$ ISO - para avaliar carga de químicos; ${ }^{4 /}$ Alvura final com carga fixa - para avaliar a branqueabilidade; ${ }^{5 /}$ Perda de rendimento depois do estágio usando equações baseadas na medição de COT - Perda de rendimento (\%) = $0,0719 * \operatorname{COT}(\mathrm{kg} \mathrm{C} / \mathrm{t}$ a.s. $)+0,9844$.

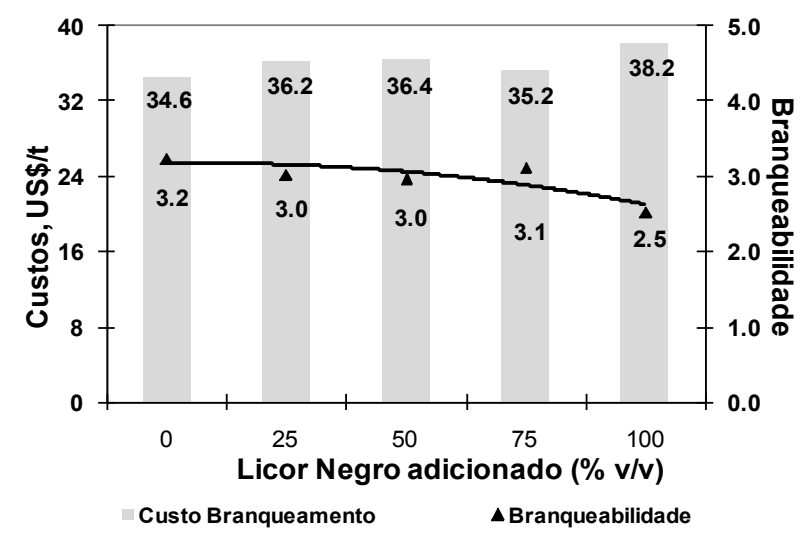

FIGURA 4: Custo de branqueamento e branqueabilidade das polpas derivadas de cozimento kraft com diferentes adições de licor negro.

FIGURE 4: Cost of bleaching and ability for bleaching of pulp from kraft pulping with different additions of black liquor.

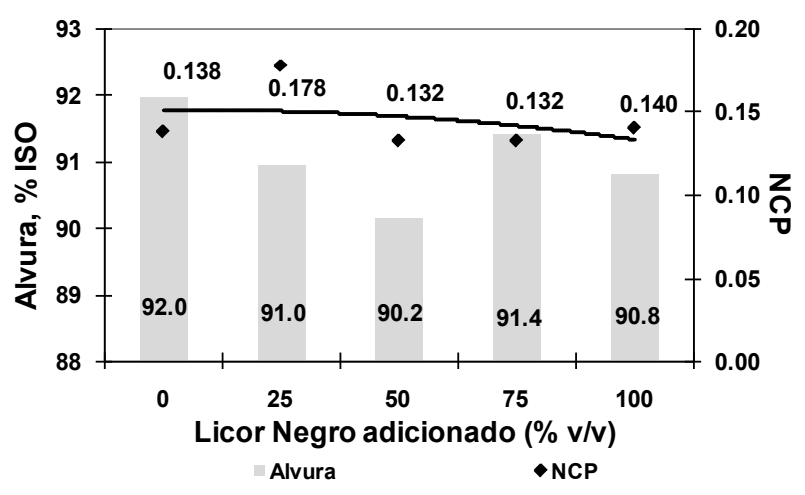

FIGURA 5: Alvura final e número de cor posterior das polpas derivadas de cozimento kraft com diferentes adições de licor negro.

FIGURE 5: Brightness and final color number after the pulps from kraft cooking with different additions of black liquor. 
Em geral, as viscosidades finais das polpas branqueadas não apresentaram diferenças significativas $\left(\mathrm{F}_{\text {calc }}-4,2\right.$ e $\left.\mathrm{F}_{\text {tab }}-5,19\right)$. Apenas uma leve diminuição é percebida à medida que se aumentou o nível de adição de licor negro aos cozimentos kraft, provavelmente devido à maior carga alcalina utilizada no cozimento e às maiores cargas de reagentes químicos no branqueamento para alcançar a alvura-objetivo (Figura 6).

\section{Comportamento das propriedades físico- mecânicas e ópticas das polpas derivadas da polpação $k r a f t$ com adição de licor negro}

As propriedades das polpas estão apresentadas em forma de gráficos, relacionados em função dos correspondentes índices de tração obtidos das polpas não refinadas e refinadas. O índice de tração foi escolhido para se relacionar graficamente com as demais propriedades, por se tratar de uma das propriedades mais exigidas para se produzir papéis para diversas finalidades. Além disso, essa propriedade é influenciada pelas variáveis dos processos usados para a sua produção que podem alterar, por exemplo, o número de ligações interfibras, a resistência das ligações e outras características importantes da estrutura dos papéis, que dependem da composição química e da característica física das polpas (SALVADOR et al., 2000).

As curvas de regressão ajustadas para índice de rasgo, em função do índice de tração para cada um dos cinco tratamentos $(0,25,50,75$ e $100 \%$ de adição de licor negro), foram comparadas com base no teste $\mathrm{F}$, e a hipótese de igualdade das cinco curvas

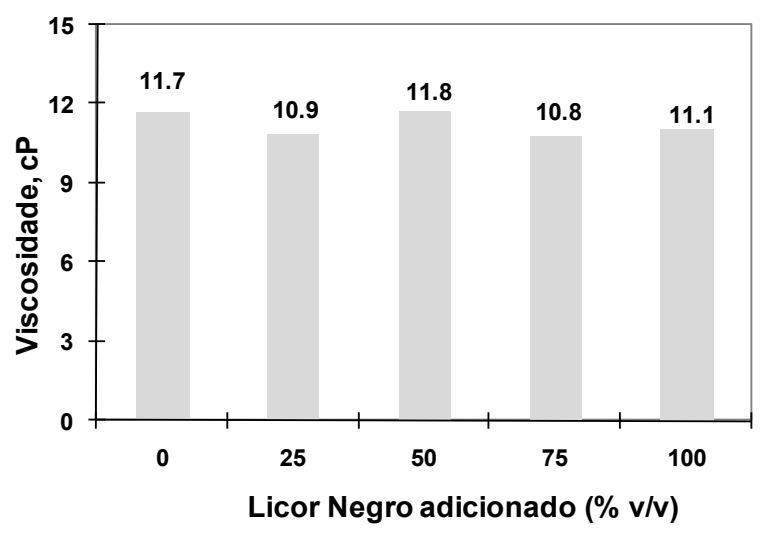

FIGURA 6: Viscosidade final das polpas oriundas de cozimento kraft com diferentes adições de licor negro.

FIGURE 6: Final viscosity of pulp from kraft cooking with different additions of black liquor. não foi rejeitada, podendo-se concluir que elas não diferem significativamente $(\mathrm{P}>0,05)$. Assim, uma curva comum pode ser usada como uma estimativa das cinco curvas envolvidas (Figura 7).

$\mathrm{O}$ índice de tração ótimo foi determinado considerando o ponto máximo da curva comum de resposta para índice de rasgo. Esse procedimento é adotado comumente nas empresas, como forma de comparação entre as curvas e escolha do melhor nível de refino, uma vez que o índice de rasgo apresenta queda acentuada para valores elevados de refino.

$\mathrm{O}$ melhor índice de rasgo foi conseguido com valores de índice de tração de aproximadamente $80 \mathrm{Nm} / \mathrm{g}$. SALVADOR et al. (2000), estudando as propriedades da celulose de fibra curta branqueada por diversas sequência de branqueamento, obtiveram valores de rasgo de aproximadamente $7 \mathrm{mNm}^{2} / \mathrm{g}$ para índice de tração de $80 \mathrm{Nm} / \mathrm{g}$. Portanto, os valores de índice de rasgo encontrados nesse trabalho ( 9 - $10 \mathrm{mNm}^{2} / \mathrm{g}$ ) podem ser considerados aceitáveis, independentemente da adição de licor negro aos cozimentos.

As curvas ajustadas para índice de tração em função do grau Schopper Riegler (resistência à drenagem), índice de estouro, capilaridade Klemm, resistência à passagem de ar, opacidade, volume específico, índice de retenção de água para cada um dos cinco tratamentos $(0,25,50,75$ e $100 \%$ de adição de licor negro), estão apresentadas na Figura 8. Com base no teste F, a hipótese de igualdade das

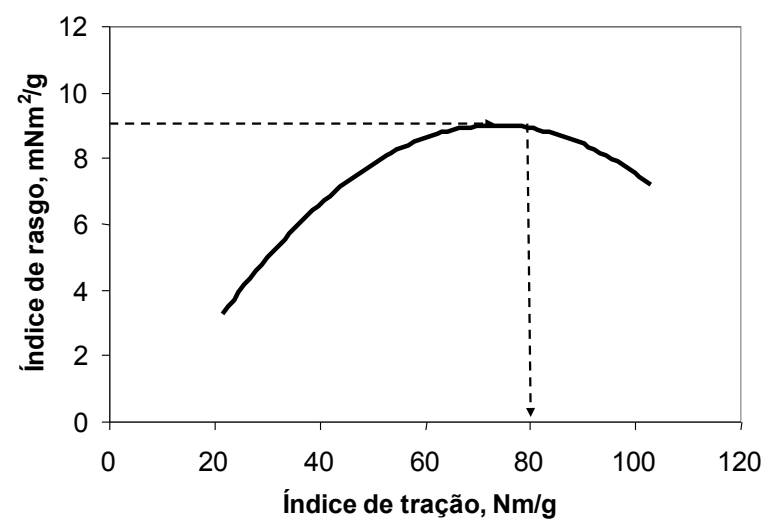

FIGURA 7: Curva comum ajustada para índice de rasgo em função do índice de tração para polpas derivadas de cozimento kraft com diferentes adições de licor negro.

FIGURE 7: Curve common set to tear index as a function of tensile index for pulp from kraft cooking with different additions of black liquor. 

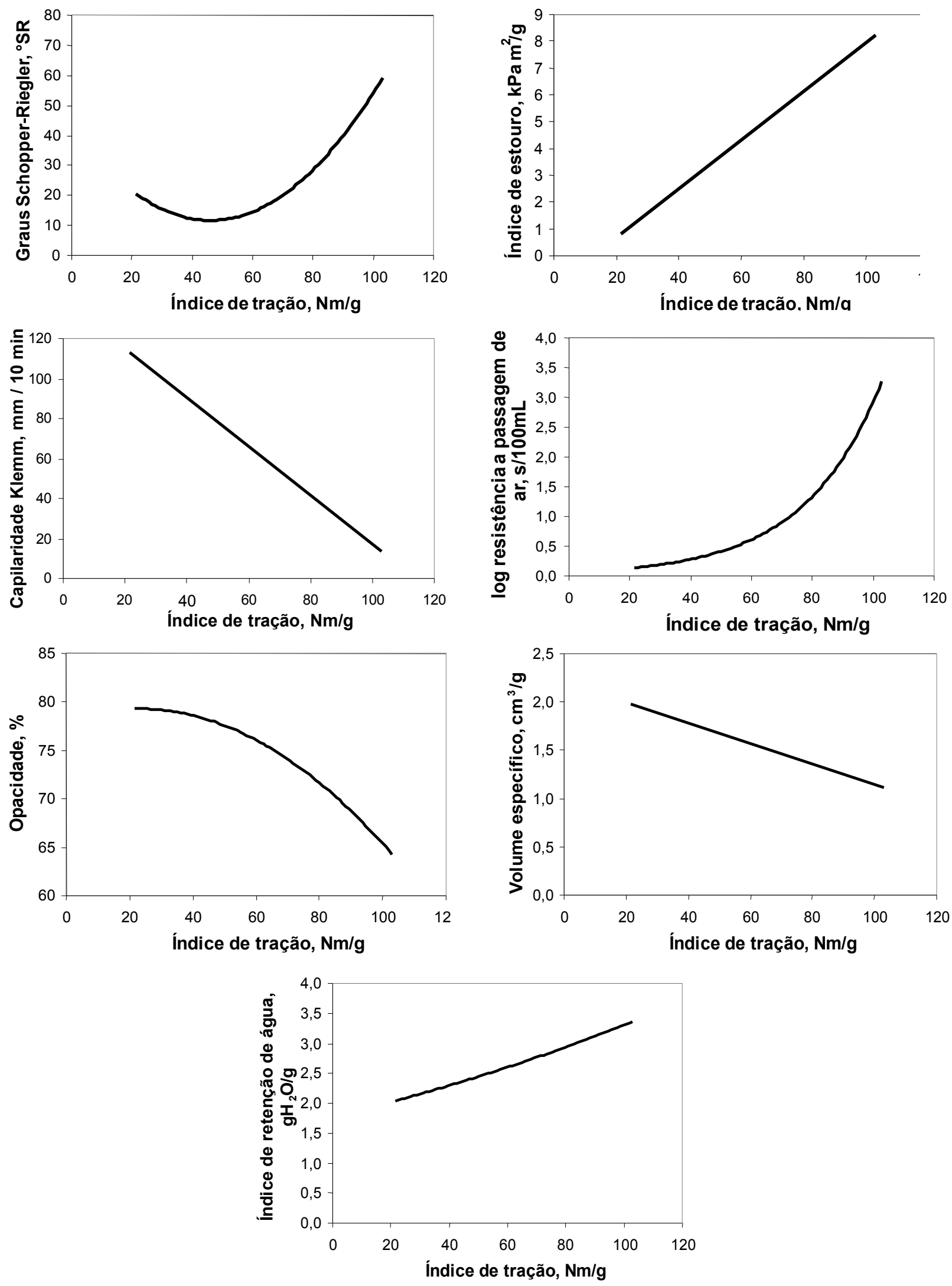

FIGURA 8: Curvas comuns ajustadas das propriedades que não apresentaram diferenças significativas polpas derivadas de cozimento kraft com diferentes adições de licor negro.

FIGURE 8: Curves common set of properties that were not significantly different - pulps from kraft cooking with different additions of black liquor. 
cinco curvas não foi rejeitada, podendo-se concluir que as cinco curvas não diferem significativamente $(\mathrm{P}>0,05)$. Assim, uma curva comum pode ser usada como estimativa das cinco curvas envolvidas para cada uma dessas propriedades avaliadas.

Salvador et al. (2000) encontraram valores de índice de estouro entre 6,4 e $8,0 \mathrm{kPam}^{2} / \mathrm{g}$, utilizando várias sequências de branqueamento, o que indica que os valores encontrados neste estudo (5,8 $6,1 \mathrm{kPam}^{2} / \mathrm{g}$ ) são ligeiramente inferiores, mas ainda praticáveis nas indústrias.

O volume específico apresentou tendência de redução com o aumento do refino, devido ao colapsamento das fibras e à maior produção de finos.

A adição de licor negro aos cozimentos não afetou a opacidade do papel. Esta propriedade óptica é importante, principalmente quando o papel é usado para fins de impressão e escrita.

Salvador et al. (2000) verificaram que a opacidade de polpas oriundas de várias sequências de branqueamento, para índice de tração de $80 \mathrm{Nm} / \mathrm{g}$, foi de aproximadamente $75 \%$. Dessa forma, os valores encontrados neste estudo $(72-73 \%)$ estão de acordo com a literatura consultada.

A única propriedade a apresentar diferença estatística foi o alongamento. Com base no teste $\mathrm{F}$, a hipótese de igualdade das cinco equações foi rejeitada, podendo-se concluir que as cinco curvas diferem significativamente $(P>0,05)$. As curvas ajustadas para índice de tração em função do alongamento, para cada um dos quatro tratamentos (25, 50, 75 e $100 \%$ de adição de licor negro), diferiram do tratamento $0 \%$ de adição de licor negro. Portanto duas curvas distintas foram plotadas na Figura 9.

Pode-se concluir, assim, que a adição de licor negro aos cozimentos não afetou de forma significativa as propriedades avaliadas nesse estudo.

\section{CONCLUSÕES}

- A eficiência e o ganho de alvura da prédeslignificação com oxigênio foram ligeiramente prejudicados pela adição de licor negro ao cozimento kraft.

- A adição de $50 \%$ de licor negro aos cozimentos kraft resultou em polpas de pior branqueabilidade, sendo a demanda de cloro ativo aumentada em 5,3 kg/tas para obtenção de alvura 92 $\%$ ISO.

- As viscosidades finais e as estabilidades

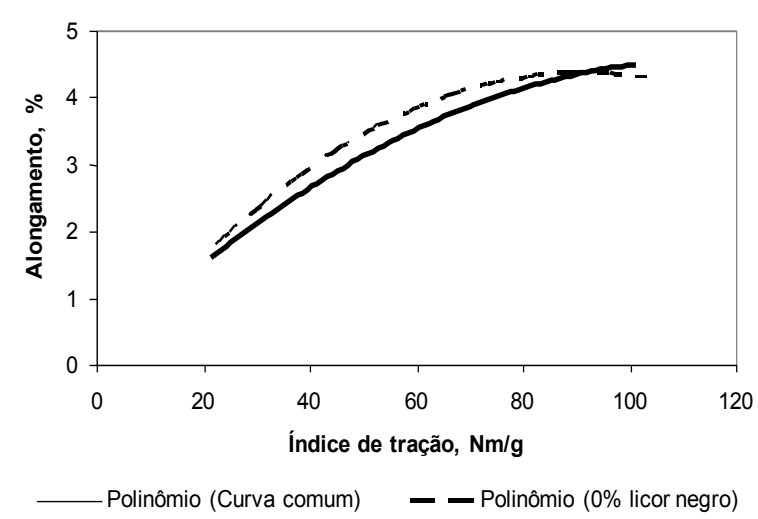

FIGURA 9: Curva comum e curva com $0 \%$ de licor negro ajustadas para alongamento em função do índice de tração para polpas derivadas de cozimento kraft com diferentes adições de licor negro.

FIGURE 9: Common curve and curve with 0\% of black liquor adjusted to elongation dependent on the traction index for pulps from kraft cooking with different additions of black liquor.

de alvura das polpas branqueadas não foram muito influenciadas pelo uso de licor negro no cozimento kraft.

- As propriedades físico-mecânicas e ópticas das polpas Kraft branqueadas não foram influenciadas, significativamente, pelo uso de licor negro no cozimento.

\section{REFERÊNCIAS BIBLIOGRÁFICAS}

BRITT, K. W. Handbook of pulp and paper technogy. 2nd ed., New York: Van Nostrand Reinhold, 1970. 723 p.

GULLICHSEN, J.; PAULAPURO, H. Papermaking science and technology. Finland: Papet Oy, 1999. 350 p. v. 3.

McDONALD, R. G. Pulp e paper manufacture: kraft Pulping. 2nd ed., New York: McGraw-hill Book Company, 1967. 725 p. v. 2

REGAZZI, A. Teste para identificar a identidade de modelos de regressão e igualdade de alguns parâmetros num modelo polinomial ortogonal. Revista Ceres, Viçosa, v. 40, n. 228, p. 176-195. 1993.

REGAZZI, A.; SILVA, C. H. O. Teste para verificar a igualdade de parâmetros e a identidade de modelos de regressão não-linear. I. Dados no delineamento inteiramente casualizado. Revista Matemática 
Estatística, São Paulo, v. 22, n. 3, p. 33-45. 2004. SALVADOR, E. et al. Efeito da deslignificação com oxigênio nas propriedades físico-mecânicas de polpas kraft. In: CONGRESSO INTERNACIONAL DE CELUlOSE E PAPEL, 2000, São Paulo.
Anais... ABTCP: São Paulo, 2000. v. 1. p. 24.

SJÖDAHL, R. Some aspects on the effects of dissolved wood components in kraft pulping. 2006.

$64 \mathrm{f}$. Tese (Doutorado em Tecnologia de Celulose)Royal Institute of Technology, Stockholm, 2006. 